\title{
Sigma- Delta Modulator Simulation and Analysis using MatLab
}

\author{
Thuneibat S. A. ${ }^{1} \&$ Ababneh M. S. ${ }^{2}$ \\ ${ }^{1}$ Department of Electrical Engineering, BAU, Jordan \\ ${ }^{2}$ Finance and Administrative Department, BAU, Jordan \\ Correspondence: Thuneibat S. A., Department of Electrical Engineering, BAU, Jordan. E-mail: \\ thuneibat@Hotmail.com
}

Received: June 29, 2012 Accepted: July 24, 2012 Online Published: August 17, 2012

doi:10.5539/cis.v5n5p76 URL: http://dx.doi.org/10.5539/cis.v5n5p76

\begin{abstract}
In addition to compatibility with VLSI technology, sigma-delta converters provide high level of reliability and functionality and reduced chip cost. Those characteristics are commonly required in the today wireless communication environment. The objective of this paper is to simulate and analyze the sigma-delta technology which proposed for the implementation in the low-digital-bandwidth voice communication. The results of simulation show the superior performance of the converter compared to the performance of more conventional implementations, such as the delta converters. Particularly, this paper is focused on simulation and comparison between sigma-delta and delta converters in terms of varying signal to noise ratio, distortion ratio and sampling structure.
\end{abstract}

Keywords: Sigma-Delta converter, Delta converter, simulation, analysis and comparison

\section{Introduction}

Sigma-Delta modulation ( $\Sigma-\Delta \mathrm{M}$ or $\Delta-\Sigma \mathrm{M})$ is a technique for converting analog signals into digital data. $\Sigma-\Delta \mathrm{M}$ is an improvement of an older Delta modulation (DM or $\Delta$-modulation). DM is an analog-to-digital modulation technique may be used mainly for a wide variety of applications, including digital telephony over the Internet (VoIP), digital wireless and $4^{\text {th }}$ generation mobile communications.

$\mathrm{DM}$ and $\Sigma-\Delta \mathrm{M}$ are types of differential pulse-code modulation (DPCM) where the difference between sample value and the previous quantized value is quantized into fixed step size $\pm \Delta$ and then encoded using 1-bit encoder, binary 1 for $+\Delta$ and binary 0 for $-\Delta$. Thus for increasing analog signal, the converter outputs 1 's and for decreasing signal, the converter outputs 0's (Schreier, 2005).

DM suffers from slope overload distortion and quantizing or granular noise, as shown in Figure 1(a). The slope overload distortion occur when the signal is increasing or decreasing with high dynamic range, that with high slope. At the other hand, granular noise takes place for a signal with low dynamic range. There are two approaches in avoiding noise and improving the performance characteristics; the first one is based on technique development, an example of such approach is the adaptive delta modulation (ADM) or continuously variable slope delta modulation (CVSD).

In ADM, $\Delta$ is variable. Rather, when multiple consecutive bits (1's or 0's) have the same increasing or decreasing direction, the encoder assumes that slope overload may occur and the $\Delta$ becomes progressively larger. Otherwise, the step size becomes gradually smaller over time. ADM reduces slope error and quantizing error at the expense of increasing the system complexity and the calculation of $\Delta$ at each sample, the number of which is very large, introduces additional time delay. This fact degrades the quality of transmitted voice, which is, as known, sensitive to delay.

The second approach is based on signal processing methods; $\Sigma-\Delta \mathrm{M}$ is an example of this approach. In $\Sigma-\Delta \mathrm{M}$, the signal is applied to an integrator, therefore the $\Sigma$ notation we put prior to $\Delta \mathrm{M}$. Both of the slope overload and granular problems are reduced with a $\Sigma-\Delta \mathrm{M}$, often to the point of insignificance. The desired output value is applied to the summing junction along with the actual output. This delta value is added to the integrator value (sigma) and applied to quantizer. The quantizer trims the data down to whatever the converter is capable of representing (Sangil, 1993).

Recently, a large number of publications deal with the issue of analysis and design of $\Sigma-\Delta \mathrm{M}$. Reference 
(Bernhard, 1988) studded the oversampled analog-to-digital converter architectures and proposed a second-order $\Sigma-\Delta \mathrm{M}$ circuit which offers a means of exchanging resolution in time for that in amplitude so as to avoid the difficulty of implementing complex precision analog circuits. In Jaykar (2011), the authors proposed a set of models which takes into account SC $\Sigma-\Delta \mathrm{M}$ nonidealities, such as sampling jitter since switching circuitry is included, kT/C noise, and operational amplifier parameters (noise, finite dc gain, finite bandwidth, slew-rate and saturation voltages). Each nonideality is modeled mathematically and their behavior is verified using different analysis in MatLAB Simulink. A new behavioral model of a second order law-pass Switched Capacitor (SC) $\Sigma$ - $\Delta \mathrm{M}$ proposed in (Abdelghani, 2011).

In this paper, we focused on the programming of $\Sigma-\Delta M$ using MatLAB apparatus for the analysis of different performance parameters and for the comparison with $\Delta \mathrm{M}$.

This paper is organized as follows: Section 2 presents the derivation of $\Sigma-\Delta \mathrm{M}$ from $\Delta \mathrm{M}$ and shows the block diagrams. Section 3 presents the developed MatLAB program. Comparison and simulation results reported in Section 4. Finally, Section 5 draws some conclusions.

\section{Derivation of Sigma-Delta Modulator from Delta Modulator}

The $\Sigma-\Delta \mathrm{M} n$ was derived from delta modulation and developed to be an extension to it. Figure 1(a) shows the block diagram of delta modulator and Figure 1(b) shows demodulator. Delta modulation is based on quantizing the difference of signal amplitude between current and previous sample using 1 bit rather than quantizing the absolute value of the signal at each sample using 8 bits as in PCM.

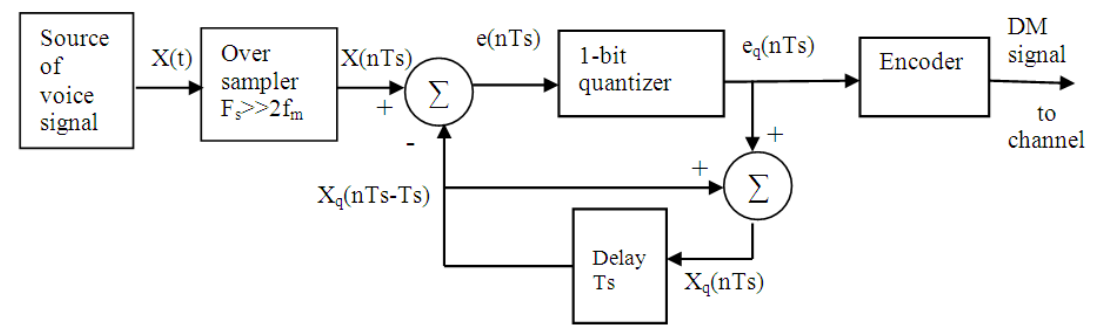

(a)

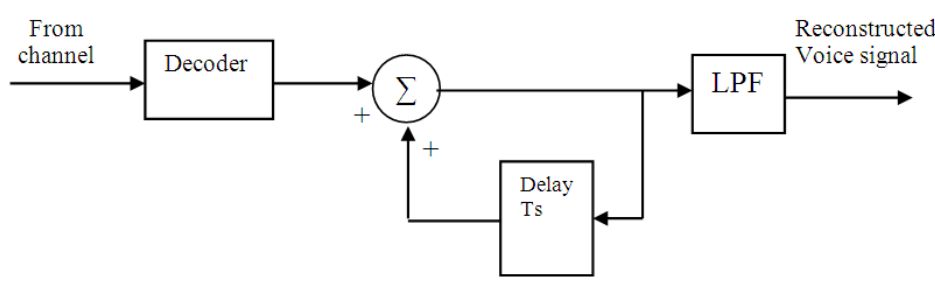

(b)

Figure 1. Delta modulator block diagram (a) modulator, (b) demodulator

$\Sigma-\Delta \mathrm{M}$ involves the use of 2 integrators for modulation and demodulation as shown in Figure 2(a) and 2(b). Since integration is a linear operation, the second integrator can be moved to the position before the modulator without altering the overall input/output characteristics. Furthermore, the two integrators in Figure 3 can be combined into a single one by the linear operation property.

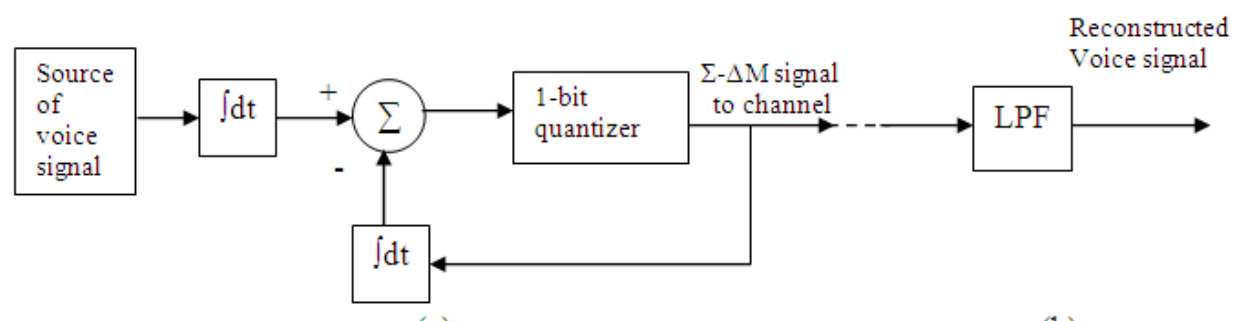

(a)

(b)

Figure 2. $\Sigma-\Delta \mathrm{M}$ block diagram (a) modulator, (b) demodulator 


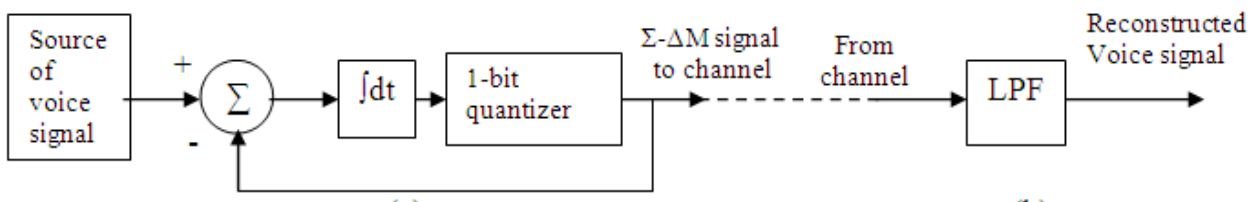

(a)

(b)

Figure 3. Simplified $\Sigma-\Delta M$ block diagram (a) modulator, (b) demodulator

\section{Communication System with Sigma-Delta Modulator}

In Section 2, we had explained the structure and block diagram of the $\Sigma$ - $\Delta \mathrm{M}$ modulator. In this section, the block diagram of a communication system using $\Sigma-\Delta \mathrm{M}$ is illustrated in Figure 4 . As the most of digital communication systems are, nowadays, going optically, the system shown hereon is implementing fiber optics as the transmission media.

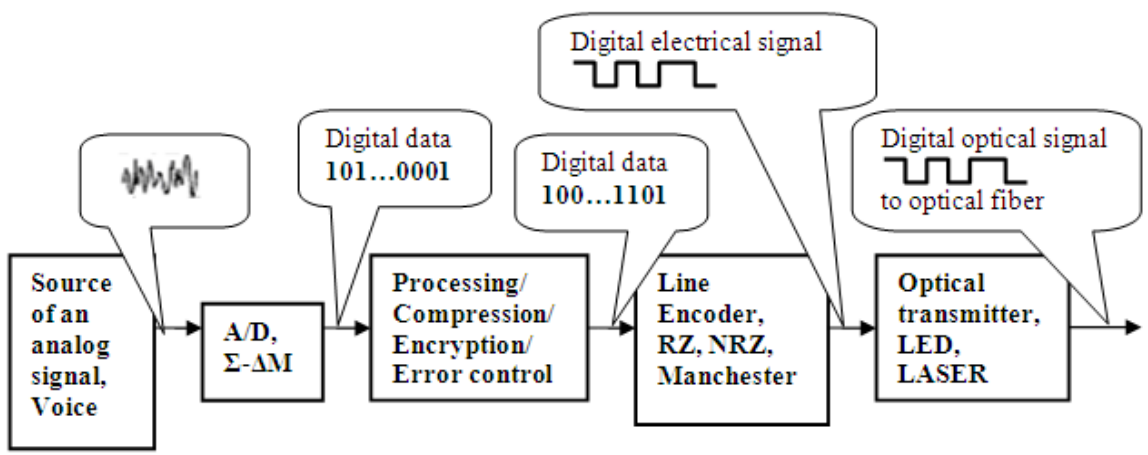

(a)

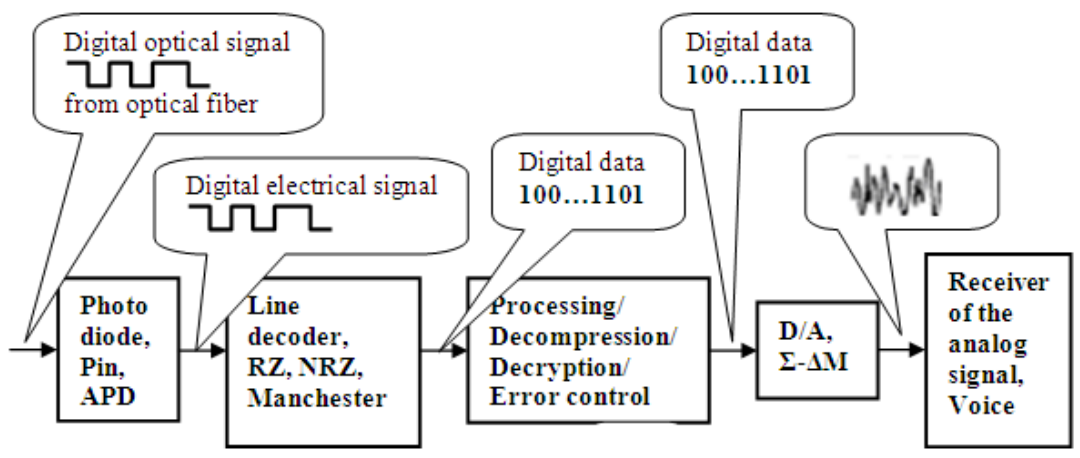

(b)

Figure 4. Digital and optical communication system block diagram (a) transmitter; (b) receiver

\section{Comparison and Simulation Results}

For the simulation and comparison of $\Sigma-\Delta \mathrm{M}$ and $\Delta \mathrm{M}$, we developed MatLAB simulation programs: the main (main.m) program and two supporting (TxDM.m) and (RxDM.m). Several runs using PC Pentium 4 had been performed and the results are presented in Figures 5 to 8. In Figure 5, (a) is the analog input signal and (b) the output of integrator, the analog signal with quick change became smoother and slower. Figure 6 presents the original and the modulated signals using (a) $\Delta \mathrm{M}$, and (b) $\Sigma-\Delta \mathrm{M}$. Figure 7 shows the message signal and the modulated at $\mathrm{SNR}=40$ using (a) $\Delta \mathrm{M}$, and (b) $\Sigma-\Delta \mathrm{M}$. In Figure 8, comparison characteristics are illustrated (a) approximate RMS error versus frequency, and (b) bit error rate versus SNR. 


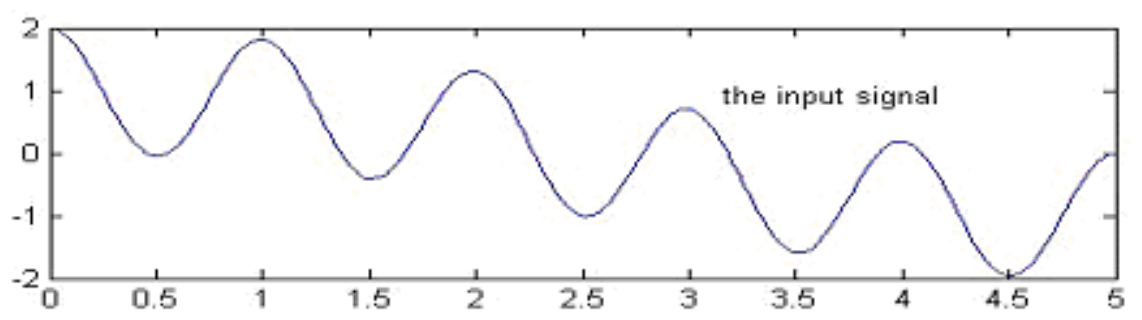

(a)

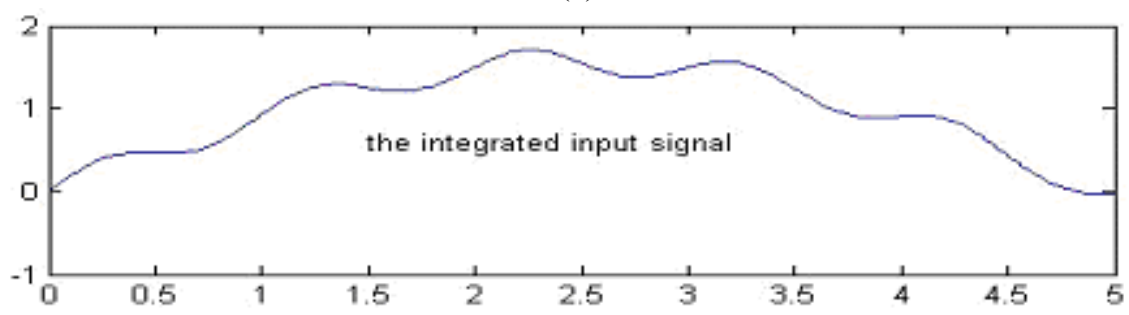

(b)

Figure 5. Integrator (a) an analog input signal; (b) integrated output

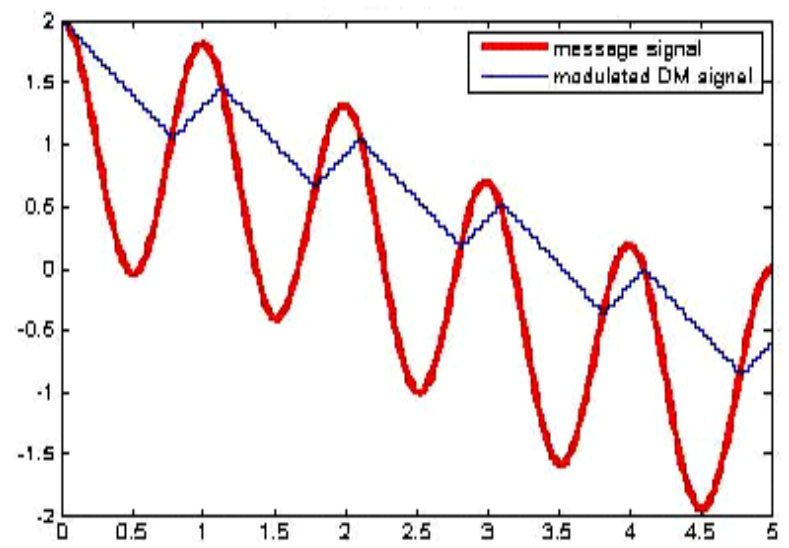

(a)

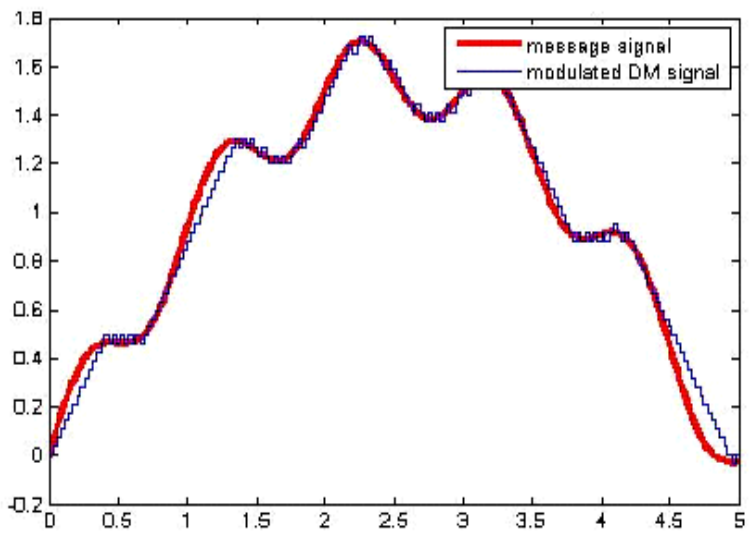

(b)

Figure 6. The message signal and the modulated using (a) $\Delta \mathrm{M}$, and (b) $\Sigma-\Delta \mathrm{M}$

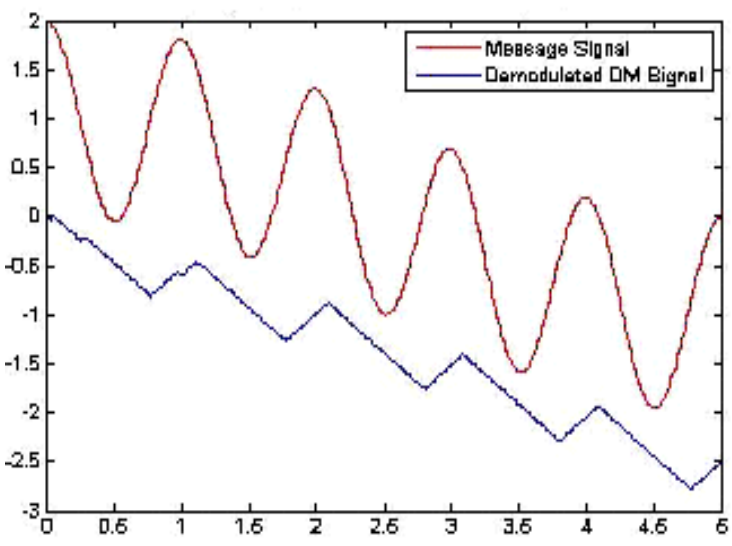

(a)

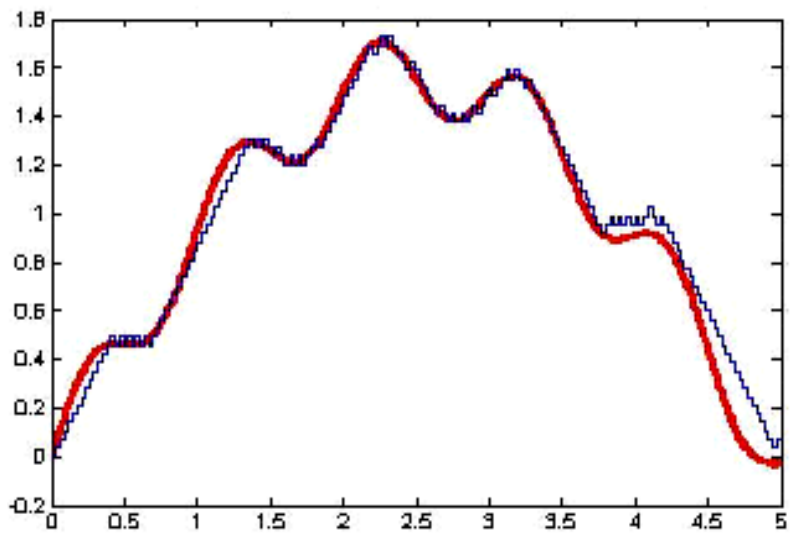

(b)

Figure 7. The message signal and the modulated at $\mathrm{SNR}=40$ using (a) $\Delta \mathrm{M}$, and (b) $\Sigma-\Delta \mathrm{M}$ 


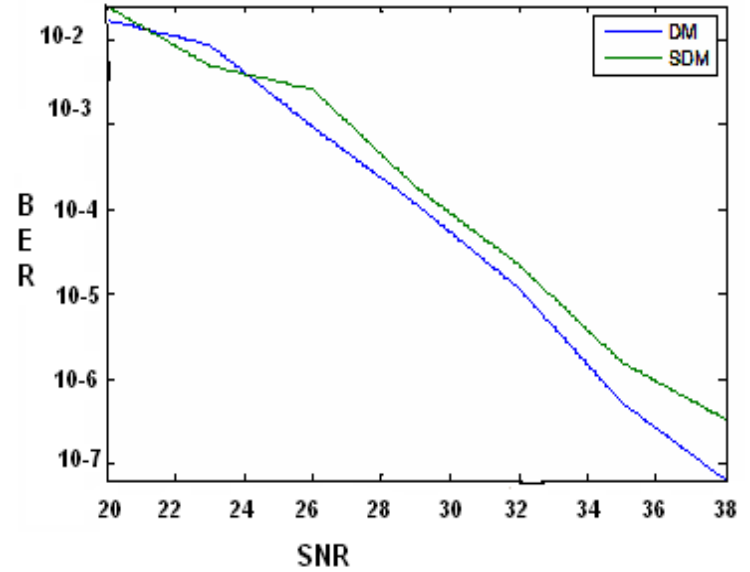

(a)

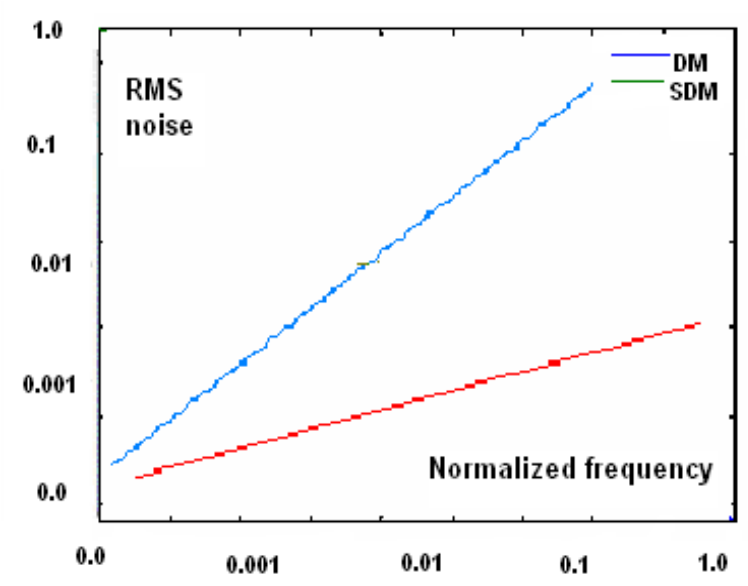

(b)

Figure 8. Comparison characteristics (a) approximate RMS error versus frequency, and (b) bit error rate versus SNR

\section{Conclusions}

$\Sigma-\Delta \mathrm{M}$ has found increasing use in audio applications over the Internet where an analog audio signal is converted into a digital audio signal which will be sent in a digital form, the modern electronic components such as analog-to-digital converters and digital-to-analog converters, frequency synthesizers, switched-mode power supplies and motor controllers. From the figures, we note the following:

- Integration in $\Sigma-\Delta \mathrm{M}$ makes signal smooth and remove the quick change, this simplifies quantization and reduce the BER, comparing with $\Delta \mathrm{M}$.

- From Figures 6 and 7, we clearly see that the modulated by $\Sigma-\Delta M$ signal is closely follow the modulating signal, rather than in $\Delta \mathrm{M}$.

- Figure 8(a) demonstrates the improvement in BER, achieved by $\Sigma-\Delta \mathrm{M}$.

- Figure 8(b) shows, may be, the only one disadvantage of $\Sigma-\Delta \mathrm{M}$. The increase of RMS error with increasing frequency, which also increased in $\Delta \mathrm{M}$ but with slope less than in $\Sigma-\Delta \mathrm{M}$.

\section{References}

Abdelghani, D., Nour-Eddine, B., Souhil, K., \& Samir, B. (2011). Modeling of a Second Order Sigma-Delta Modulator with Imperfections. International Journal on Electrical Engineering and Informatics, 3(2), 327-332.

Bernhard, E. B., \& Wooley, B. A. (1988). The Design of Sigma-Delta Modulation Analog-to-Digital Converters. IEEE Journal of Solid-State Circuits, 23(6), 1298-1308. http://dx.doi.org/10.1109/4.90025

Jaykar, S., Palsodkar, P., \& Dakhole, P. (2011). Modeling of Sigma-Delta Modulator Non-Idealities in MATLAB/SIMULINK. Communication Systems and Network Technologies (CSNT), 2011 International Conference on, 525-530.

Sangil. P. (1993). Motorola Digital Signal Processors: Principles of Sigma-Delta Modulation for Analog-to-Digital Converters. Motorola.

Schreier, R., \& Temes, G. C. (2005). Understanding Delta-Sigma Data Converters. New York: IEEE Press. 